\title{
A single-session Acceptance and Commitment Therapy intervention targeting shame in people experiencing homelessness: A randomized multiple baseline design
}

\author{
Varsha Eswara Murthy, Ian Hussey, \& Louise McHugh
}

\begin{abstract}
Background and Objectives: Individuals experiencing homelessness are frequently discriminated against, such as through reduced employment opportunities and interpersonal rejection, creating a vicious cycle that sustains homelessness. These external conditions of stigma often result in individuals developing an internal sense of self-stigma and intense shame, which can result in negative psychological and behavioral consequences. Psychological intervention may contribute to disrupting this vicious cycle. Acceptance and Commitment Therapy (ACT) has been demonstrated to treat shame and self-stigma in other domains, but never homelessness. Methods: We developed a single-session intervention suitable for this population, and tested it within a randomized multiple-baseline Single Case Experimental Design (SCED). Shame and experiential avoidance were measured daily via SMS messages for eightynine days in 9 individuals experiencing homelessness. Results: SCED analysis and meta-analysis across participants indicated that the intervention showed significant improvements in experiential avoidance and shame both within and between participants. Limitations: Limitations include a lack of assessment of overt behavior change and the results of single case studies may have limited generalizability. Conclusions: Results demonstrate the efficacy of a brief ACT intervention in reducing shame and experiential avoidance in a homeless population, as well as the general benefits of single case design methodologies when working with populations that are difficult to reach or recruit from.
\end{abstract}

Research has highlighted that homeless populations have generally higher health needs, including a higher prevalence of severe mental illness than the housed community (Lowry, 1990; Timms \& Balázs, 1997). This is also true in Ireland (O'Carroll \& O'Reilly, 2008). However, less than a third of people experiencing homelessness and mental health problems receive treatment (Scott, 1993), putting the population at severely increased risk of a multitude of mental and behavioral health disorders including posttraumatic stress disorder, substance abuse, and increased suicide attempts (O'Connor, 2005). In addition to this, the stigma associated with experiencing homelessness can lead to self-stigma and intense shame (Kidd, 2007), resulting in negative psychological and behavioral impacts, such as avoidance of treatment (Scambler, 1998; Starr,
Campbell, \& Herrick, 2002; Thornicroft, 2008), avoidance of contact with others (Dorahy, 2010; Zavaleta, 2015) and diminished help-seeking (Clement et al., 2015; Collins \& Barker, 2009). This often leads to some sort of social sanction or devaluation, reducing the probability of the person being hired, or being trusted as a parent, friend, or partner (Johnstone, Jetten, Dingle, Parsell, \& Walter, 2015; Milburn, Ayala, Rice, Batterham, \& Rotheram-Borus, 2006).

Shame has been argued to be the emotional core of the experience of stigma and tends to involve strong or literalized beliefs of being flawed or unlovable (Luoma \& Platt, 2015). In addition, the effects of enacted stigma - the emotional and behavioral barriers erected by individuals in response to perceived or experienced stigma - may also serve to obstruct access to opportunities (Luoma, Kohlenberg, Hayes, \& 
Fletcher, 2012). Shame and self-stigma have been argued to both point to indictments of one's character, rather than descriptions of either one's behavior or indeed the wider structural inequalities that gave rise to that behavior (Luoma \& Platt, 2015). As such, while self-stigma and shame are the psychological consequences of external, structural and material systems, once behavioral repertoires of self-stigma and shame are established in an individual they may participate in the maintenance of broader maladaptive behaviors. These psychological variables present targets for psychotherapeutic intervention.

Given that individuals who are experiencing homelessness are by definition an often-transient population, brief interventions that can be delivered in a small number of sessions are desirable to achieve treatment coverage. Despite this, evidence on brief interventions targeting psychological well-being with the homeless population is sparse. While Assertive Community Treatment and case-management interventions have been proven moderately effective in improving mental and physical health outcomes for homeless populations (O'Connor, 2005), the resources and expertise required to run such interventions are typically unavailable to most sectors and communities. When they are employed they are time consuming, leaving large portions of this population untreated. Elsewhere, brief psychological interventions with the homeless population has demonstrated promising efficacy (Peterson, Baer, Wells, Ginzler, \& Garrett, 2006). However, these interventions often target specific sub-populations, choose to focus on one aspect of recovery such as substance abuse and do not address comorbidity of illness (FEANTSA \& Mental Health Europe, 2009). In our opinion, in order to meet the psychological wellbeing needs of those experiencing homelessness, there remains an unfulfilled need for psychological interventions that are (a) tailored for use with individuals experiencing homelessness, (b) brief, (c) cost-effective, and (d) easily trainable.

Acceptance and Commitment Therapy (ACT) demonstrates several properties that make it a good candidate here. ACT is a cognitive and behavioral therapy that aims to specifically reduce avoidance of internal experiences, including shame and critical thoughts, in order to foster a satisfying life guided by one's freely chosen values in the face of external challenges (Hayes, Strosahl, \& Wilson, 1999). It has been previously demonstrated to be effective in reducing shame and self-stigma in numerous domains, including substance, body satisfaction, and sexual orientation (Luoma, Kohlenberg, Hayes, Bunting, \& Rye, 2008; Luoma et al., 2012; Luoma et al., 2007; Yadavaia \& Hayes, 2012). This makes ACT a good candidate for our current goals. It has also been demonstrated to be effective with populations of varying levels of comorbidity and complex mental health needs, which are common in individuals experiencing homelessness (Batten \& Hayes, 2005; Lang et al., 2017; Peterson \& Zettle, 2009). However, to date, no empirical work has examined the efficacy of ACT-based approaches with homeless populations.

Conceptually, ACT interventions frequently aim to alter clients' relationships with their unwanted negative thoughts, emotions, and feelings, rather than the content of the thoughts, emotions, and feelings themselves (Hayes, Strosahl, et al., 1999). There is increasing evidence that thoughts may not need to be changed for CBT to be effective (Longmore \& Worrell, 2007). Moreover, attempts to suppress or control unwanted internal experiences can be counterproductive (e.g. Campbell-Sills, Barlow, Brown, \& Hofmann, 2006; Tran \& Rimes, 2017). This has been argued to be particularly the case in cases where suffering is irreducible or be linked to contextual and structural inequalities that are outside of an individuals' control, such as the experience of homelessness (Campbell \& Deacon, 2006). Within $\mathrm{ACT}$, one form of maladaptive relationship within one's unwanted internal experiences is referred to as experiential avoidance (Bond et al., 2011). On the basis of their dual theoretical relevance within this population, this study therefore tested a single-session ACT-based intervention for shame and experiential avoidance within a homeless population.

\section{Method}

\section{Design}

We report how we determined our sample size, all data exclusions, all manipulations, and all measures in the study (Nelson, Simmons, \& Simonsohn, 2012). The study followed a randomized-multiple baseline Single Case Experimental Design (SCED). By treating each individual as a separate experiment, collecting data from multiple time points for each, and then meta analyzing across participants, SCEDs provide high quality evidence for the effectiveness of an intervention using a far smaller number of participants than a Randomized Controlled Trial (RCT). This property of SCEDs made them particularly suitable to the current research, given this difficult to reach population. In a recent review of clinical SCED research, Duan, Kravitz and Schmid (2013; see also Deaton \& Cartwright, 2018) noted that an additional benefit of SCED over group-based designs is their focus on variability in and experimental control over individuals' behavior rather than group means. Specifically, treatments that have been shown to be beneficial on average may nonetheless deliver an uneven mix of risks, ineffectiveness, and benefits to 
individuals (i.e., suffer from heterogeneity of treatment effects).

SCEDs are also of practical utility because they require fewer participants than an RCT. In many contexts, including the current one, it would simply not be feasible to collect data from a sufficiently large sample for statistical analyses to have sufficient power. By adopting SCED we overcame a key practical barrier that may explain the relative dearth of existing literature in this area. Research within populations that are difficult to reach or recruit from, such as individuals experiencing homelessness, may therefore benefit from SCED designs.

A randomized multiple-baseline SCED design was therefore implemented across participants. The independent variable for each participant was the prepost intervention phase, and the dependent variables were self-reported experiential avoidance and shame. Assessments were made at the same total number of timepoints for all participants $(n=89)$. Following recommended practices, the specific day in which a participant received the ACT protocol was randomized across a 5 to 7 -day period (Kratochwill \& Levin, 2010). Depending on when the intervention was delivered, baseline data was collected over a period of 7 to 53 days, and post-intervention data collection lasted for between 35 to 81 days. All randomizations were conducted using the web-based tool Research Randomizer (Urbaniak \& Plous, 2013).

\section{Participants}

The sample was recruited as follows: the lead researcher contacted service managers in two charities that catered to issues around homelessness, who subsequently communicated details of the research project to key workers. Key workers were then informed on the nature of the study, the inclusions and exclusion criteria, and the must remain contactable during interviews by the lead researcher. Inclusion criteria were currently experiencing homelessness or experience of homelessness in the last 6-months. Exclusion criteria were current symptoms of psychosis or suicidality, severe cognitive impairment or learning difficulties, or a below conversational level of English. If the key worker felt that the study was suitable for implementation with their clients, they then identified potential participants who they considered to meet the criteria and discussed participation with them who approached individuals they deemed to meet the criteria. If the client wished to participate, the lead researcher then contacted them either by phone or in person to arrange a meeting to discuss the study and obtain written informed consent.

Twenty meetings were arranged, with 15 agreeing to participate in an appointment set up to collect baseline data. Twelve participants attended baseline collection and 3 participants subsequently dropped out post-baseline (see Figure 1). The remaining participants were also called or reminded in person by the key worker the day before and the day of data collection and the intervention to remind the participant of the time and location of the both. The final sample consisted of 9 participants, each of whom were either currently experiencing homelessness or had experienced homelessness in the past 6-months. A majority of participants were male ( 7 men, 2 women) and young adults $\left(M_{\text {age }}=23.2 ; S D=2.2\right.$, range 20 to 26). They reported having experienced homelessness for between 9 and 84 months $(M=45.1, S D=27.0)$. Five reported no current substance use, one reported alcohol use, and two reported multi-drug use (including heroin, methadone, benzodiazepines, and cannabis), and data was missing for one. Seven identified as straight, one as bisexual, and one as gay. Participants had between 8 and 17 years of education $(M=12.6, \quad S D=2.7)$. Seven reported being unemployed, one as employed, and one a full-time student.

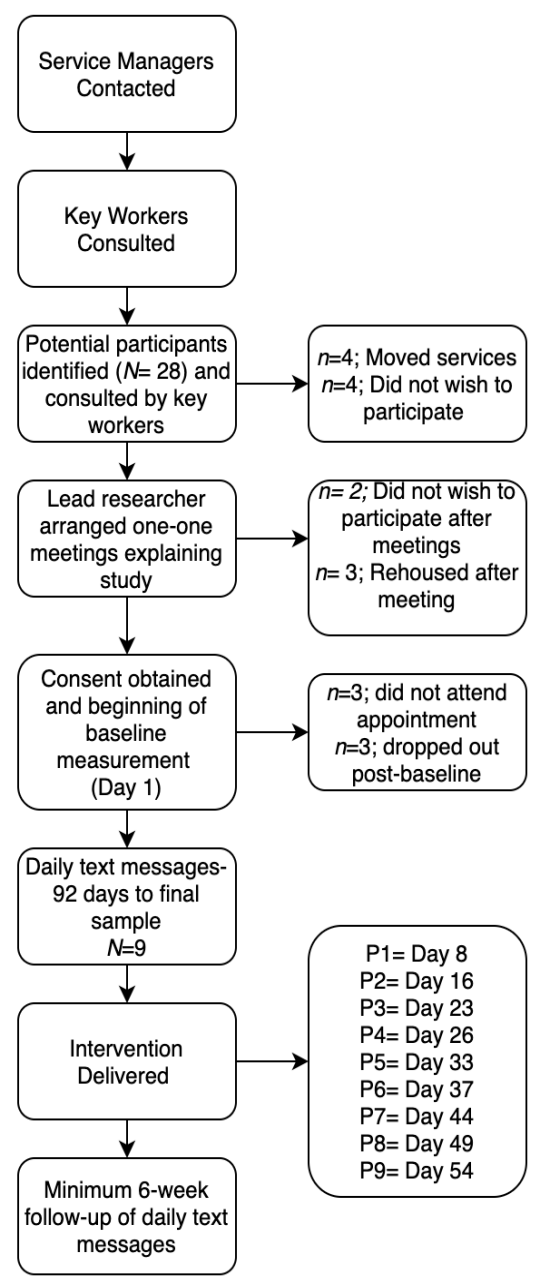

Figure 1. Flowchart of study progression. 
Table 1. Phases of the ACT-based intervention.

\begin{tabular}{|c|c|c|}
\hline Phase & Aims & Intervention \\
\hline $\begin{array}{l}\text { Defining Stigma and Shame } \\
\text { (15 mins) }\end{array}$ & $\begin{array}{l}\text { To identify and clarify the difference } \\
\text { between enacted and self-stigma }\end{array}$ & $\begin{array}{l}\text { - Identify stereotypes about the } \\
\text { homeless } \\
\text { - Identify a situation where it has } \\
\text { - limited them } \\
\text { Identify a time and how they judge } \\
\text { themselves }\end{array}$ \\
\hline $\begin{array}{l}\text { Creative Hopelessness } \\
\text { (10 minutes) }\end{array}$ & Introducing control as the problem & 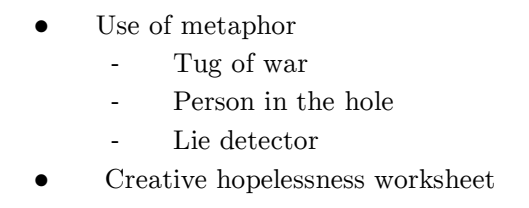 \\
\hline $\begin{array}{l}\text { Defusion training } \\
\text { (15 minutes) }\end{array}$ & $\begin{array}{l}\text { Noticing thoughts as barriers to } \\
\text { action }\end{array}$ & $\begin{array}{l}\text { - } \quad \text { Leaves on the stream } \\
\text { - } \quad \text { 'I'm having the thought that...' } \\
\text { - } \quad \text { Content on cards }\end{array}$ \\
\hline $\begin{array}{l}\text { Acceptance } \\
\text { (10 minutes) }\end{array}$ & $\begin{array}{l}\text { Developing willingness as an } \\
\text { alternative to suppression }\end{array}$ & - $\quad$ Physicalizing mindfulness \\
\hline $\begin{array}{l}\text { Values Clarification } \\
\text { (10 minutes) }\end{array}$ & $\begin{array}{l}\text { To clarify what matters to clients in } \\
\text { order to identify a hierarchical } \\
\text { motivator to sustain behavior } \\
\text { change }\end{array}$ & $\begin{array}{l}\text { - Distinguish between values as } \\
\text { direction vs a goal } \\
\text { - Values card sort }\end{array}$ \\
\hline $\begin{array}{l}\text { Committed action and } \\
\text { Rounding Up } \\
\text { (15 minutes) }\end{array}$ & $\begin{array}{l}\text { Encourage workable behavior } \\
\text { change }\end{array}$ & - $\quad$ ACT Matrix \\
\hline
\end{tabular}

Note: Full intervention protocol and script available at osf.io/hy24v

\section{Measures}

The dependent variables were two single-item measures of shame and experiential avoidance. These items were selected and developed based on their acceptability and validity prior to the study through an informal assessment of brevity, face validity, and acceptability in consultation with both domain experts and the target population. First, a number of candidate shame items were considered from an established psychometric self-report measure of shame: The Internalized Shame Scale (Cook, 2001). Five candidate experiential avoidance items were created by independent reviewers who have expertise in ACT and existing ACT-related measures of experiential avoidance (e.g., CompACT Scale: Francis, Dawson, \& Golijani-Moghaddam, 2016). These items were then trialed via an online survey with 49 service users and service providers to assess their clarity and interpretability, as well as their perceived face validity. These items therefore prioritized (a) brevity and (b) interpretability across a wide range of reading or impairment levels that may have been encountered in the sample. Two items were selected to be completed by participants in the study. Instructions were "On a scale from 1-10, 1 being not at all and 10 being very much so, please indicate how you are feeling right now in relation to the questions below". The items were "I feel like I am not good enough" (shame) and "Have your thoughts and feelings blocked you today?" (experiential avoidance).

\section{Single-session ACT Intervention}

The intervention consisted of six phases (see Table 1 for summary; full intervention protocol and

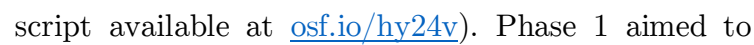
define, identify and clarify different types of stigma and identify how external perceptions or stereotypes about individuals experiencing homelessness can lead one to internalize these stereotypes and can limit one's own behavior. Phase 2 provided psycho-education on how engaging in experiential avoidance of internal experiences such as unwanted thoughts and feelings related to the self can lead to moving away from a valued life in the long-term. A worksheet was then completed with the service user in order to demonstrate how engaging in actions to avoid unwanted internal experiences in the short-term has led to unwanted outcomes in their lives long-term.

In Phase 3, the service user was introduced to the concept of defusion, as used within ACT. As a physical metaphor the participant was asked to write a shamebased thought, they had about themselves on a card and hold it straight in front of their faces, in order to demonstrate how you can sometimes be blinded and blinkered by unwanted thoughts. The service user was then instructed to hold the card far away from the face and with a considerable amount of strength, this was done in order to generate an intense physical 
experience to demonstrate the effort it takes to try and push-away or get rid of difficult thoughts. The service user was then instructed to lay the card on their lap and notice how they can engage with the world even though the thought is still present. The participant was then guided through either the leaves on the stream exercise or the "I'm having the thought" exercise in order to create space between the self and unwanted thoughts.

Phase 4 involved an acceptance-based eyes closed guided meditation known as physicalizing mindfulness. Participants were guided through a series of steps in order to identify physical attributes of an unwanted difficult feeling. They were then encouraged to notice the space between themselves and the experience, as a method of creating a sense of 'self as context' (Hayes, Strosahl, et al., 1999). Finally, they were instructed to hold the feeling lightly within themselves.

Phase 5 included an identifying and recognizing values exercise. The values card sort asked participants to sort a though 40 cards with values written on them into 3 piles labelled as 'not important to me', 'somewhat important to me', and 'very important to me'. They were asked to pick 5 cards from the 'very important' pile and discuss why those values were important to them.

Phase 6 involved bringing together all the different elements that had been covered in the previous phases. The ACT Matrix (Polk, Schoendorff, Webster, \& Olaz, 2016), a paper-based exercise, was used to help identify unwanted internal experiences surrounding a chosen life domain, recognize what unhelpful strategies the participant may be engaging in to avoid those unwanted internal experiences, and finally identify a series of both short and long-term goals to reach a valued direction in their chosen life domain.

\section{Procedure}

The study was conducted at two homelessness shelters in inner-city Dublin, Ireland. Ethical approval was granted by the local Institutional Review Board. Individuals referred by their key worker who elected to participate provided written informed consent. Both single-item questions were sent to participants each day via SMS message, who also responded via SMS. The timing of these SMS messages was customized for each participant to suit their schedule, and were typically sent between the hours of 9:00 AM and 9:00 PM. SMS messages were deemed the most suitable method on the basis that individuals in this population were likely to have access to a SMS-capable mobile phone. Baseline measurement started on the same day for all participants. After the baseline phase, the intervention was delivered in a single-session, delivered to each participant individually in a one-toone format delivered by the first and third authors. The interventionist was randomized between these two individuals for each participant (one senior, one in training). At the end of the session the participant was given a booklet with psychoeducational information, brief exercises, and audios to complete if they so wished.

\section{Treatment fidelity}

Interventions were audio recorded and observed by three members of the lead researcher's lab. Treatment fidelity was assessed post-intervention by 3 independent reviewers: a peer-reviewed ACT trainer and two $\mathrm{PhD}$ researchers who have received between three to five years of training in ACT. This involved at least 2 reviewers listening to the same 10-minute recording from each session and noting the ACT processes they can identify within the 10-minute recording. The degree of agreement between the independent raters of treatment fidelity was good, $x=$ .82 (Cohen, 1960). Therapists also recorded what exercises were used to teach each of the ACT processes for each session. These records were then assessed by the independent reviewers to assess protocol adherence (e.g., were all of the ACT processes taught and were the exercises in the protocol used).

\section{Analytic strategy}

Analyses were conducted using the $\mathrm{R}$ package SCED (Hussey, 2019), which is designed to provide simple and robust visualization, analysis, and metaanalysis of A-B SCED data. Following the long tradition of visual analysis of SCED data (see Lane \& Gast, 2014 for an accessible primer), raw data along with regression trend lines, median values per phase, and median absolute deviation of the scores within each phase were first plotted (see Figure 2). This allows for the visual assessment of between-phase difference, baseline trends, and variability in each phase. Data were also subjected to quantitative analysis on the basis that research has also shown visual inspection to have low inter-rater reliability (Ottenbacher, 1990; Park, Marascuilo, \& GaylordRoss, 1990; Ximenes, Manolov, Solanas, \& Quera, 2009), and conclusions were drawn primarily from the quantitative analyses. SCED data routinely violates parametric assumptions; as such the SCED package focuses on the use of nonparametric statistics, including hypothesis testing via permutation tests (see Nichols \& Holmes, 2002; Onghena \& Edgington, 1994) and bootstrapping of a nonparametric effect size: the probability of superiority (Ruscio, 2008; Ruscio \& Mullen, 2012). This metric represents the probability that a randomly chosen data point after intervention is superior to a randomly chosen data before intervention. This is annotated $P(\mathrm{~A}<\mathrm{B})$. 

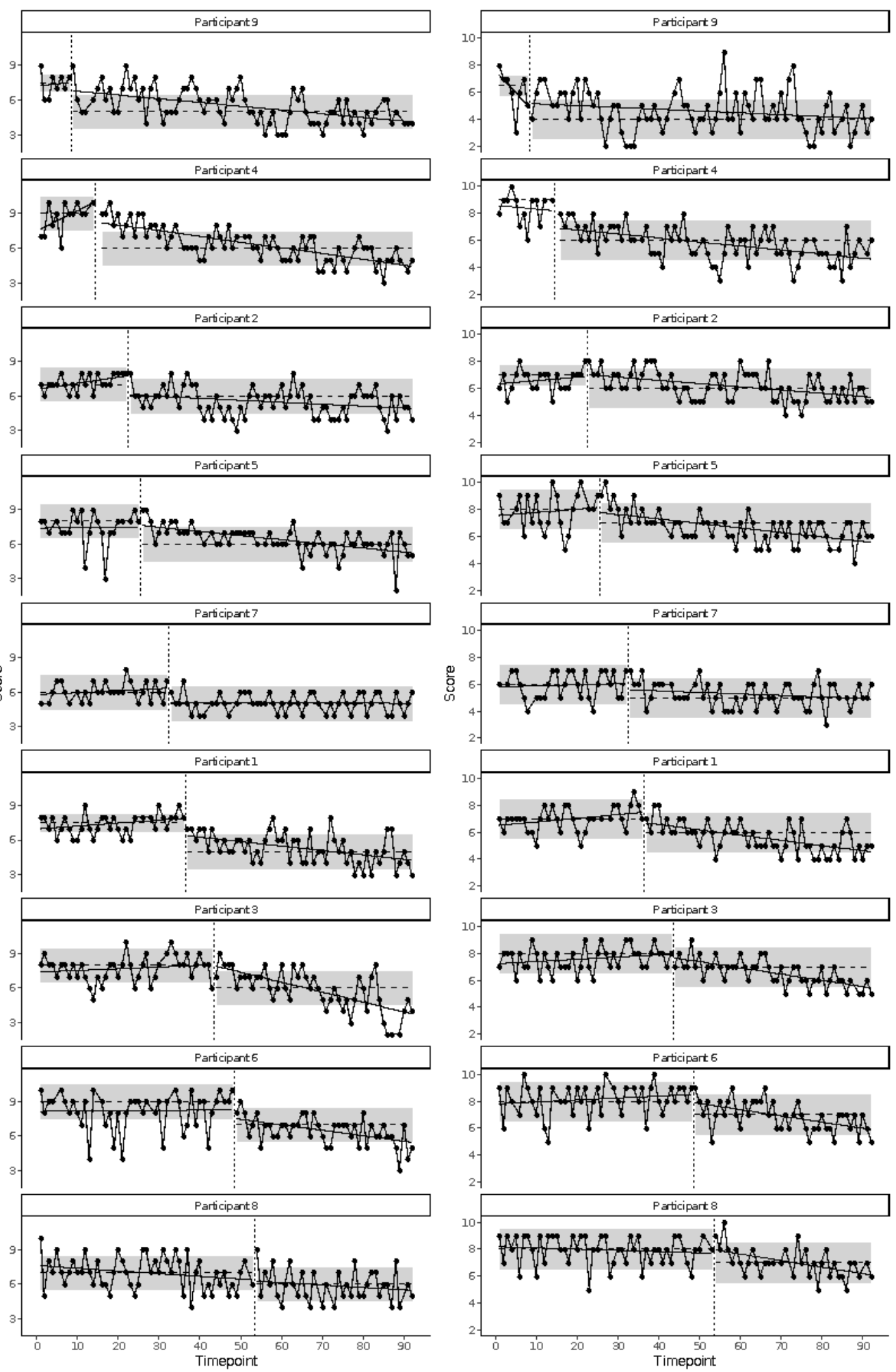

Figure 2. SCED data for each participant. Left and right panels refer to the experiential avoidance and shame measures respectively. Dotted vertical lines refer to the point at which the intervention was delivered. Shaded areas represent the one median absolute deviation from the median. Dashed horizontal lines represent the median. Solid lines represent OLS regression lines to diagnose within-phase trends. 


\begin{tabular}{|c|c|c|c|c|c|c|c|}
\hline $\begin{array}{l}\text { Participant } 1 \\
\text { Participant } 2 \\
\text { Participant } 3 \\
\text { Participant } 4 \\
\text { Participant } 5 \\
\text { Participant } 6 \\
\text { Participant } 7 \\
\text { Participant } 8 \\
\text { Participant } 9\end{array}$ & & & 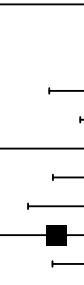 & 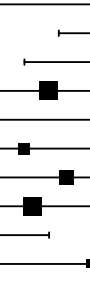 & $\begin{array}{l}\vec{a} \\
- \\
-\end{array}$ & & $\begin{array}{l}0.89[0.82,0.94] \\
0.87[0.78,0.93] \\
0.80[0.71,0.88] \\
0.87[0.74,0.94] \\
0.78[0.63,0.88] \\
0.82[0.71,0.90] \\
0.79[0.68,0.87] \\
0.71[0.60,0.81] \\
0.85[0.71,0.93]\end{array}$ \\
\hline \multirow[t]{3}{*}{ RE Model $\left(I^{2}=38.3 \%, H^{2}=1.6\right.$} & & & & - & & & $0.82[0.78,0.86]$ \\
\hline & 1 & $T$ & $T$ & $T$ & $T$ & $\neg$ & \\
\hline & 0.50 & 0.60 & 0.70 & 0.80 & 0.90 & 1.00 & \\
\hline
\end{tabular}

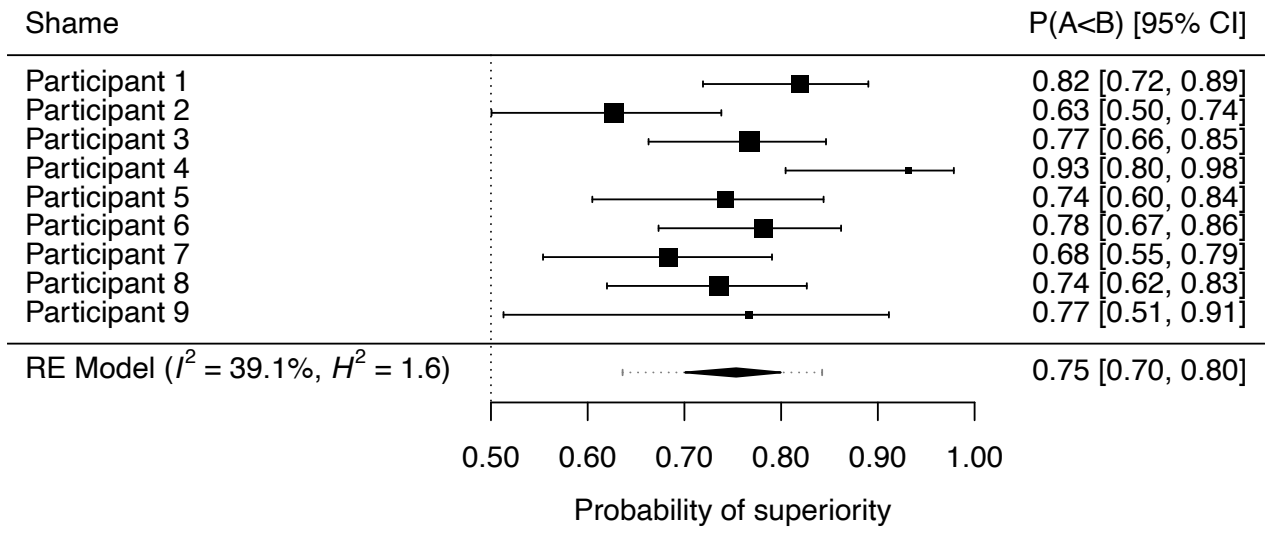

Figure 3. SCED meta-analysis forest plots.

This effect size metric has been recommended for SCED research on the basis of its high interpretability even for non-experts, robustness, and on the basis that it represents an extension of an effect size that has seen much use in SCED work: Percentage of Nonoverlapping Difference (Parker \& Vannest, 2009). The probability of superiority has been referred by multiple other names the Probabilistic Index (Acion, Peterson, Temple, \& Arndt, 2006; Thas, De Neve, Clement, \& Ottoy, 2012), Non-overlap All Pairs (Parker \& Vannest, 2009), the Dominance Statistic, and others, as well as being closely related to the Common Language Effect Size (McGraw \& Wong, 1992). Hedges' $g$ values (Hedges, 1981) - that is, Cohen's $d$ effect sizes with small sample size bias corrections are also reported for the sake of reader familiarity. However, we note that its parametric assumptions are frequently violated by SCED data. median differences between the before and after intervention phases are also reported as a robust unstandardized effect size metric.
Finally, standardized effect sizes were metaanalyzed across participants. For this the SCED package makes use of the established metafor package (Viechtbauer, 2010). Specifically, the SCED metaanalysis function fit random effects models using Maximum Likelihood estimation. Meta-analysis models were fit for both the probability of superiority and Hedges' $g$ values from each participant. In the case of the probability of superiority, estimates were transformed to odds prior to fitting, and results of the meta-analysis models were converted back to probabilities. Following standard meta analytic practices, models were used to calculate $95 \%$ confidence intervals on the meta effect size, metrics of between-subjects heterogeneity in the effect size $\left(\tau^{2}\right.$, $I^{2}$, and $H^{2}$ ), and $95 \%$ credibility intervals on the effect size (i.e., the range of mean effect sizes likely to be observed given observed heterogeneity). Sensitivity analyses were carried out by reincluding participants who demonstrated trends at baseline and examining their impact on results. 
Table 2. Results of the SCED analyses and meta-analyses.

\begin{tabular}{|c|c|c|c|c|c|c|}
\hline Outcome & Participant & Trend & $p$ & Median & Ruscio's A & Hedges' $g$ \\
\hline \multicolumn{7}{|c|}{ Experiential Avoidance } \\
\hline & 1 & 0.28 & $<.0001$ & -2.5 & $.89[.94, .82]$ & $1.76[2.24,1.35]$ \\
\hline & 2 & $0.45^{*}$ & $<.0001$ & -1 & $.87[.93, .79]$ & $1.50[1.96,1.05]$ \\
\hline & 3 & 0.16 & $<.0001$ & -2 & $.80[.88, .70]$ & $1.22[1.59,0.81]$ \\
\hline & 4 & $0.48^{*}$ & $<.0001$ & -3 & $.87[.95, .74]$ & $1.61[2.18,0.96]$ \\
\hline & 5 & 0.10 & .0014 & -2 & $.78[.87, .62]$ & $0.83[1.43,0.16]$ \\
\hline & 6 & 0.06 & $<.0001$ & -2 & $.82[.90, .71]$ & $1.20[1.75,0.65]$ \\
\hline & 7 & 0.17 & $<.0001$ & -1 & $.79[.86, .67]$ & $1.23[1.71,0.73]$ \\
\hline & 8 & -0.23 & .0004 & -1 & $.71[.81, .60]$ & $0.80[1.24,0.36]$ \\
\hline & 9 & 0.08 & .0006 & -2.5 & $.85[.93, .72]$ & $1.33[1.85,0.73]$ \\
\hline & Meta & - & $<.0001$ & -2 & $.82[.78, .86]$ & $1.28[1.06,1.49]$ \\
\hline & Sensitivity & - & $<.0001$ & -2 & $.81[.76, .85]$ & $1.21[0.96,1.46]$ \\
\hline \multicolumn{7}{|l|}{ Shame } \\
\hline & 1 & 0.29 & $<.0001$ & -1 & $.82[.89, .72]$ & $1.32[1.79,0.84]$ \\
\hline & 2 & 0.18 & .0887 & -1 & $.63[.74, .50]$ & $0.43[0.83,0.02]$ \\
\hline & 3 & 0.21 & $<.0001$ & -1 & $.77[.85, .66]$ & $1.08[1.49,0.61]$ \\
\hline & 4 & -0.07 & $<.0001$ & -3 & $.93[.98, .81]$ & $2.11[2.68,1.45]$ \\
\hline & 5 & 0.14 & $<.0001$ & -1 & $.74[.84, .60]$ & $0.98[1.52,0.37]$ \\
\hline & 6 & 0.13 & $<.0001$ & -1 & $.78[.86, .67]$ & $1.10[1.59,0.61]$ \\
\hline & 7 & 0.08 & .0024 & -1 & $.68[.79, .56]$ & $0.72[1.23,0.26]$ \\
\hline & 8 & -0.18 & .0001 & -1 & $.74[.83, .62]$ & $0.85[1.33,0.34]$ \\
\hline & 9 & $-0.62^{*}$ & .0104 & -2.5 & $.77[.90, .47]$ & $0.99[1.68,0.12]$ \\
\hline & Meta & - & $<.0001$ & -1 & $.75[.70, .80]$ & $1.04[0.75,1.33]$ \\
\hline & Sensitivity & - & $<.0001$ & -1 & $.75[.70, .80]$ & $1.05[0.73,1.37]$ \\
\hline
\end{tabular}

Notes: Trends refer to ordinal regression beta values between score and time point prior to intervention; $p=$ permuted $p$ values for the difference between phases; median = median difference between phases; $P(\mathrm{~A}<\mathrm{B})=$ the probability of superiority between phases (i.e., the probability that a randomly chosen data point after intervention is better than a randomly chosen data point before intervention); Hedges' $g=$ standardized difference between phases; Meta = meta-analyzed effect size; Sensitivity $=$ meta-analyzed effect size after excluding participants with baseline trends $>0.3 ; *=$ participants in sensitivity analysis; square brackets contain $95 \%$ Confidence Intervals.

\section{Results}

All data and code are available on the Open Science Framework (osf.io/hy24v). Data for both the experiential avoidance and shame outcome variables is present in Figure 2. This plot orders participants by the timing of the intervention in order to illustrate the impact of the intervention across the multiple baselines. Visual inspection of the plots indicated that change in both experiential avoidance and shame was likely attributable to the intervention: scores appeared to improve as a function of the intervention. This pattern was then examined quantitatively (see Table 2 for results, and Figure 3 for meta-analysis forest plots).
9 of 9 participants (100\%) demonstrated significant decreases in experiential avoidance as a function of the intervention. Meta-analysis across participants suggested that the intervention produced large decreases in experiential avoidance, $P(\mathrm{~A}<\mathrm{B})=$ $.82,95 \%$ CI $[.78, .86], 95 \%$ CR $[.72, .89], p<.0001$, Hedges' $g=1.28,95 \%$ CI $[1.06,1.49], 95 \%$ CR [0.82, 1.74]. Metrics of heterogeneity in the probability of superiority between participants were non-significant and of moderate size, $Q(8)=12.5, p=.13, \tau^{2}=0.07$, $I^{2}=38.28, H^{2}=1.62$.

8 of 9 participants (89\%) demonstrated significant decreases in shame as a function of the intervention. Meta-analysis suggested that the intervention produced large decreases in shame, $P(\mathrm{~A}<\mathrm{B})=.75$, 
$95 \%$ CI $[.70, .80], 95 \%$ CR $[.64, .84], p<.0001$, Hedges' $g=1.04,95 \%$ CI $[0.75,1.33], 95 \%$ CR [0.28, 1.80]. Metrics of heterogeneity in the probability of superiority between participants were again nonsignificant and of moderate size, $Q(8)=14.93, p=$ $.061, \tau^{2}=0.06, I^{2}=39.09, H^{2}=1.64$.

Sensitivity analyses were also performed following the SCED package recommendations. These excluded participants that had trends at baseline, which could represent natural improvement unrelated to the intervention (i.e., ordinal regression slope between score and time point $> \pm 0.3$ ). For the experiential avoidance measure two participants were excluded and results were robust to these exclusions, $P(\mathrm{~A}<\mathrm{B})$ $=.81,95 \%$ CI $[.76, .85], 95 \%$ CR $[.70, .88]$, Hedges' $g$ $=1.21,95 \%$ CI $[0.96,1.46], 95 \%$ CR $[0.70,1.71], p<$ .0001 , heterogeneity $Q(6)=9.45, p=.15, \tau^{2}=0.06$, $I^{2}=38, H^{2}=1.61$. For the shame measure, one participant was excluded for baseline trends and results were robust to these exclusions, $P(\mathrm{~A}<\mathrm{B})=.75$, $95 \%$ CI $[.70, .80], 95 \%$ CR [.63, .85], Hedges' $g=1.05$, $95 \%$ CI $[0.73,1.37], 95 \%$ CR [0.22, 1.88], heterogeneity $Q(7)=14.89, p=.037, \tau^{2}=0.07, I^{2}=45.52, H^{2}=$ 1.84 .

In summary, at the participant level, 9 of 9 showed significant improvements on the experiential avoidance measure, and 8 of 9 showed significant improvement on the shame measure. In meta-analyses across participants, results demonstrated that the intervention led to decreases in both experiential avoidance and shame that were of large standardized effect size. These results were robust to sensitivity analyses in which we excluded participants who demonstrated trends or excessive variation at baseline. With regard to treatment heterogeneity, on the one hand, (a) significant improvement was observed in almost all individuals (i.e., rather than being driven by a subset), (b) metrics of heterogeneity were nonsignificant (with one exception in the sensitivity analyses), and (c) the meta effect sizes of both the meta analyses' credibility intervals as well as confidence intervals excluded zero (i.e., estimates of the mean effect size likely to be observed even in light of observed heterogeneity still excluded the null effect). On the other hand, metrics of heterogeneity (i.e., $I^{2}$ and $H^{2}$ ) were of moderate size, possibly suggesting that heterogeneity was poorly estimated within the available sample size. Therefore, there remains some uncertainty around whether there is heterogeneity between participants that is greater than what would be expected due to sampling variation alone. If this were to be shown to be the case in future research, this may suggest that other variables influence the magnitude of intervention's effect (e.g., individual differences between participants, protocol adherence, treatment adherence, etc.).

\section{Discussion}

The single session ACT-based intervention for individuals experiencing homelessness was shown to reduce stigma and experiential avoidance. Results from SCED analyses and the meta-analyses across participants demonstrated that these reductions were demonstrated both within and between individuals, and were of large size (all probability of superiority $>$ .75 ; all Hedges' $g>1.04$ ). To our knowledge, this is the first study to assess the efficacy of ACT-based interventions for shame in a population of individuals experiencing homelessness.

Meta-analytic estimates of treatment effects suggested that the intervention was effective despite heterogeneity between participants (i.e., all credibility intervals excluded zero). This heterogeneity, which may have been as a result of demographic heterogeneity between participants, suggests that the findings may be generalizability. However, further studies are of course needed given the small sample size. The within-participant stability of effects over a minimum 6 -week period indicates that even a brief intervention can produce desirable outcomes and that changes were maintained for at least several weeks. The brevity of the intervention should also be noted, given that brevity is often cited as a key feature for interventions sought within homeless services.

These findings are consistent with previous research supporting the use of ACT for the treatment of shame and stigma within other domains, such as alcohol and drug use (Luoma et al., 2012), obesity (Lillis, Hayes, Bunting, \& Masuda, 2009), and sexuality (Yadavaia \& Hayes, 2012). ACT, more generally, may therefore be an effective approach for reducing shame and self-stigma in a variety of marginalized populations.

One strength of this study is its use of randomized multiple-baseline SCED. Compared to the gold standard Randomized Control Trial (Woolfolk, 2015), this required fewer participants, as every participant acts as their own control. In the unstable and unpredictable homeless sector, it is a challenge to recruit and retain the sample sizes necessary to produce meaningful results via RCT. While RCTs remain to be held as a gold standard for many, the use of SCED here made this research question feasible where it would not otherwise have been so. Single-case experimental designs have also been argued to better understandings of intra-individual processes and efficacy, given that group averages can often obscure important individual differences (Barlow, Nock, \& Hersen, 2009; Hayes, Barlow, \& Nelson-Gray, 1999). Separately, the collection of self-report in real-time or 
at specified sampling points daily (i.e., a form of Ecological Momentary Assessment: Trull \& EbnerPriemer, 2014), benefits from increased ecological validity. First, data collection happens in the natural context of the occurrence of those thoughts, feelings and behavior, and second, measurement has reduced reliance on retrospective recall and therefore reduced bias.

This study also benefited from the use of multiple therapists in the delivery of the intervention, who also differed in their level of experience. This may help increase the generalizability of the findings, and suggest that favorable outcomes can be achieved by therapists with varying levels of training. The inclusion of a protocol adherence check, through interrater evaluation, indicated that the intervention was implemented with high levels of fidelity (i.e. as designed and intended to be implemented, Webb, DeRubeis, \& Barber, 2010). Future studies could choose to include therapist competency checks to further enhance the integrity of treatment implementation and reduce the risk of type III errors.

A number of important limitations should be noted. First, the use of single-item outcome measures of shame and experiential avoidance may limit generalizability of outcomes. The decision to employ single-item measures was based on feedback from service users and service providers in the homeless sector who suggested that engagement with the study would be minimal if the burden placed on service users was high, especially given the daily assessments. We attempted to mitigate this threat to validity through an assessment of their structural validity. Nonetheless, both shame and experiential avoidance are multifaceted constructs, and therefore these items are therefore unlikely to capture the full breadth of these multifaceted constructs (Gámez et al., 2011; Tangney \& Dearing, 2003).

Second, although the sample size was adequate for SCED methodology given the high number of assessment points provided by each participant, questions remain about the generalizability of the findings to comparable populations. We note that the sample was diverse in some respect (sexuality, education, duration of experience of homelessness, substance abuse), however it was relatively homogenous in others (e.g., age: most in this study were young adults). Importantly, for pragmatic reasons, this study excluded individuals with the most severe mental health issues (e.g., those experiencing psychosis) and those with other comorbid issues (e.g., learning disabilities). Due to the nature of participation in the study, it is possible that only higher-functioning individuals were referred by key workers to participate in the study. This may present another form of selection bias. The acceptability and efficacy of this ACT-based protocol is therefore unknown within the subpopulations with more severe mental health issues or low functioning.

Third, while the follow-up period of up to 6-weeks was adequate, questions remain around whether benefits are maintained on the longer timescales. Of course, there are many practical and feasibility barriers to long term studies given the transient nature of the homeless population, given that individuals over time may no longer be accessing or affiliated with the service they were initially recruited from.

Finally, while shame and experiential avoidance have theoretical importance within our understanding of stigma and the ACT treatment model, changes in overt behavior are of ultimate interest. Direct assessment of some of these behaviors or outcomes of interest may be possible in future work, such as changes in substance use, self-harm, or service engagement. Psychological intervention on the selfstigma involved in the experience of homelessness must of course be supplemented within intervention and support at many other levels in order to remediate homelessness itself, including intervention on material conditions themselves (e.g., Stergiopoulos et al., 2019).

In conclusion, this work involved three key features and developments. First, single-item measures of shame and experiential avoidance were developed, and their face validity, acceptability, and structural validity was assessed. Second, we developed a brief ACT-based intervention aimed at decreasing shame and experiential avoidance in individuals experiencing homelessness. The form and content of this intervention were tailored to suit the needs of working with this specific population, and multiple efforts were made to make materials accessible so that others might use and refine this protocol. Third, the use of Single Case Experimental Design, using the aforementioned single-item measures and delivered through SMS messages, allowed us to study assess the efficacy of this protocol within a population of individuals experiencing homelessness, a population can be difficult to reach in sufficient numbers to run traditional RCTs. Results demonstrated that the protocol is effective in reducing both shame and experiential avoidance. Perhaps equally importantly, the freely available materials we provide serve to maximize the potential for replications, extensions, and clinical use.

\section{Funding}

This work was supported by an Irish Research Council grant GOIP/2018/1165 to VM and Ghent University grant $01 \mathrm{P} 05517$ to $\mathrm{IH}$.

\section{Conflict of Interest}

The authors declare they have no conflicts of interest. 


\section{References}

Acion, L., Peterson, J. J., Temple, S., \& Arndt, S. (2006). Probabilistic index: an intuitive nonparametric approach to measuring the size of treatment effects. Statistics in Medicine, 25(4), 591-602. https://doi.org/10.1002/sim.2256

Barlow, D. H., Nock, M., \& Hersen, M. (2009). Single case experimental designs: Strategies for studying behavior for change (3rd Ed.). Allyn and Bacon

Batten, S. V., \& Hayes, S. C. (2005). Acceptance and commitment therapy in the treatment of comorbid substance abuse and post-traumatic stress disorder: A case study. Clinical Case Studies, 4(3), 246-262. https://doi.org/10.1177\%2F1534650103259689

Bond, F. W., Hayes, S. C., Baer, R. A., Carpenter, K. M., Guenole, N., Orcutt, H. K., Waltz, T., \& Zettle, R. D. (2011). Preliminary psychometric properties of the Acceptance and Action Questionnaire-II: A revised measure of psychological inflexibility and experiential avoidance. Behavior Therapy, 42(4), 676-688. https://doi.org/10.1016/j.beth.2011.03.007

Campbell, C., \& Deacon, H. (2006). Unravelling the contexts of stigma: from internalization to resistance to change. Journal of Community \& Applied Social Psychology, 16(6), 411-417. https://doi.org/10.1002/casp.901

Campbell-Sills, L., Barlow, D. H., Brown, T. A., \& Hofmann, S. G. (2006). Effects of suppression and acceptance on emotional responses of individuals with anxiety and mood disorders. Behavior Research and Therapy, 44(9), 1251-1263. http://doi.org/10.1016/j.brat.2005.10.001

Clement, S., Schauman, O., Graham, T., Maggioni, F., Evans-Lacko, S., Bezborodovs, N., Morgan, C., Rüsch, N., Brown, J. S. L., \& Thornicroft, G. (2015). What is the impact of mental healthrelated stigma on help-seeking? A systematic review of quantitative and qualitative studies. Psychological Medicine, 45(1), 11-27. https://doi.org/10.1017/S0033291714000129

Cohen, J. (1960). A coefficient of agreement for nominal scales. Educational and Psychological Measurement, 20(1), 37-46. https://doi.org/10.1177/001316446002000104

Collins, P., \& Barker, C. (2009). Psychological helpseeking in homeless adolescents. International Journal of Social Psychiatry, 55(4), 372-384. https://doi.org/10.1177/0020764008094430

Cook, D. R. (2001). Internalized Shame Scale: Technical manual. Multi-Health Systems.
Deaton, A., \& Cartwright, N. (2018). Understanding and misunderstanding randomized controlled trials. Social Science \& Medicine, 210, 2-21. https://doi.org/10.1016/j.socscimed.2017.12.005

Dorahy, M. J. (2010). The impact of dissociation, shame, and guilt on interpersonal relationships in chronically traumatized individuals: A pilot study. Journal of Traumatic Stress, 23(5), 653656. https://doi.org/10.1002/jts.20564

Duan, N., Kravitz, R. L., \& Schmid, C. H. (2013). Single-patient (n-of-1) trials: a pragmatic clinical decision methodology for patient-centered comparative effectiveness research. Journal of Clinical Epidemiology, 66(8 0), S21-S28. https://doi.org/10.1016/j.jclinepi.2013.04.006

FEANTSA \& Mental Health Europe (2009). Joint statement in homelessness and mental health: Addressed to the newly elected Members of European Parliament. http://www.mhesme. org/assets/files/Common\%20statement\%20FEA NTSA \%20MHE\%20.pdf

Francis, A. W., Dawson, D. L., \& GolijaniMoghaddam, N. (2016). The development and validation of the Comprehensive assessment of Acceptance and Commitment Therapy processes (CompACT). Journal of Contextual Behavioral Science, 5(3), 134-145. https://doi.org/10.1016/j.jcbs.2016.05.003

Gámez, W., Chmielewski, M., Kotov, R., Ruggero, C., \& Watson, D. (2011). Development of a measure of experiential avoidance: The Multidimensional Experiential Avoidance Questionnaire. Psychological Assessment, 23(3), 692-713. https://doi.org/10.1037/a0023242

Hayes, S. C., Barlow, D. H., \& Nelson-Gray, R. O. (1999). The scientist practitioner: Research and accountability in the age of managed care (2nd Ed.). Allyn and Bacon.

Hayes, S. C., Strosahl, K., \& Wilson, K. G. (1999). Acceptance and commitment therapy: Understanding and treating human suffering. Guilford.

Hedges, L. V. (1981). Distribution Theory for Glass's Estimator of Effect size and Related Estimators. Journal of Educational Statistics, 6(2), 107-128. https://doi.org/10.3102/10769986006002107

Hussey, I. (2019). The SCED package for R: Simple and robust visualization, analysis, and metaanalysis of A-B Single Case Experimental Design data. https://github.com/ianhussey/SCED

Johnstone, M., Jetten, J., Dingle, G. A., Parsell, C., \& Walter, Z. C. (2015). Discrimination and wellbeing amongst the homeless: the role of multiple group membership. Frontiers in Psychology, 6, 739. https://doi.org/10.3389/fpsyg.2015.00739 
Kidd, S. A. (2007). Youth homelessness and social stigma. Journal of Youth and

Adolescence, 36(3), 291-299.

https://doi.org/10.1007/s10964-006-9100-3

Kratochwill, T. R., \& Levin, J. R. (2010). Enhancing the scientific credibility of single-case intervention research: Randomization to the rescue. Psychological Methods, 15(2), 124. https://doi.org/10.1037/a0017736

Lane, J. D., \& Gast, D. L. (2014). Visual analysis in single case experimental design studies: Brief review and guidelines. Neuropsychological Rehabilitation, 24(3-4), 445-463. https://doi.org/10.1080/09602011.2013.815636

Lang, A. J., Schnurr, P. P., Jain, S., He, F., Walser, R. D., Bolton, E., Benedek, D. M., Norman, S. B., Sylvers, P., Flashman, L., Strauss, J., Raman, R., \& Chard, K. M. (2017). Randomized controlled trial of acceptance and commitment therapy for distress and impairment in $\mathrm{OEF} / \mathrm{OIF} / \mathrm{OND}$ veterans. Psychological Trauma: Theory, Research, Practice, and Policy, 9(S1), 74. https://doi.org/10.1037/tra0000127

Lillis, J., Hayes, S. C., Bunting, K., \& Masuda, A. (2009). Teaching acceptance and mindfulness to improve the lives of the obese: A preliminary test of a theoretical model. Annals of Behavioral Medicine, 37(1), 58-69.

http://doi.org/10.1007/s12160-009-9083-x

Longmore, R. J., \& Worrell, M. (2007). Do we need to challenge thoughts in cognitive behavior therapy? Clinical Psychology Review, 27(2), 173187. https://doi.org/10.1016/j.cpr.2006.08.001

Lowry, S. (1990). Housing and health: health and homelessness. BMJ: British Medical

Journal, 300(6716), 32. https://doi.org/10.1136/bmj.300.6716.32

Luoma, J. B., Kohlenberg, B. S., Hayes, S. C., Bunting, K., \& Rye, A. K. (2008). Reducing selfstigma in substance abuse through acceptance and commitment therapy: Model, manual development, and pilot outcomes. Addiction Research \& Theory, 16(2), 149-165. https://doi.org/10.1080/16066350701850295

Luoma, J. B., Kohlenberg, B. S., Hayes, S. C., \& Fletcher, L. (2012). Slow and steady wins the race: A randomized clinical trial of acceptance and commitment therapy targeting shame in substance use disorders. Journal of Consulting and Clinical Psychology, 80(1), 43. https://doi.org/10.1037/a0026070

Luoma, J. B., \& Platt, M. G. (2015). Shame, selfcriticism, self-stigma, and compassion in acceptance and commitment therapy. Current
Opinion in Psychology, 2, 97-101.

https://doi.org/10.1016/j.copsyc.2014.12.016

Luoma, J. B., Twohig, M. P., Waltz, T., Hayes, S.

C., Roget, N., Padilla, M., \& Fisher, G. (2007).

An investigation of stigma in individuals receiving treatment for substance

abuse. Addictive Behaviors, 32(7), 1331-1346.

https://doi.org/10.1016/j.addbeh.2006.09.008

McGraw, K. O., \& Wong, S. (1992). A common language effect size statistic. Psychological Bulletin, 111(2), 361.

https://doi.org/10.1037/0033-2909.111.2.361

Milburn, N. G., Ayala, G., Rice, E., Batterham, P., \& Rotheram-Borus, M. J. (2006). Discrimination and exiting homelessness among homeless adolescents. Cultural Diversity and Ethnic Minority Psychology, 12(4), 658. https://doi.org/10.1037/1099-9809.12.4.658

Nelson, L. D., Simmons, J. P., \& Simonsohn, U. (2012). Let's publish fewer papers. Psychological Inquiry, 23(3), 291-293.

https://doi.org/10.1080/1047840X.2012.705245

Nichols, T. E., \& Holmes, A. P. (2002).

Nonparametric permutation tests for functional neuroimaging: A primer with examples. Human Brain Mapping, 15(1), 1-25.

https://doi.org/10.1002/hbm.1058

O'Carroll, A., \& O'Reilly, F. (2008). Health of the homeless in Dublin: has anything changed in the context of Ireland's economic boom? European Journal of public Health, 18(5), 448-453. https://doi.org/10.1093/eurpub/ckn038

O'Connor, J. (2005). Between the street and the consulting room: The role of therapeutic frame in working with homeless clients. European Journal of Psychotherapy, Counselling and Health, 7(4), 217-233. https://doi.org/10.1080/13642530500367597

Onghena, P., \& Edgington, E. S. (1994). Randomization tests for restricted alternating treatments designs. Behavior Research and Therapy, 32(7), 783-786. https://doi.org/10.1016/0005-7967(94)90036-1

Ottenbacher, K. J. (1990). Visual inspection of single-subject data: an empirical analysis. Mental Retardation, 28(5), 283-290.

Park, H.-S., Marascuilo, L., \& Gaylord-Ross, R. (1990). Visual Inspection and Statistical Analysis in Single-Case Designs. The Journal of Experimental Education, 58(4), 311-320. https://doi.org/10.1080/00220973.1990.10806545

Parker, R. I., \& Vannest, K. (2009). An Improved Effect Size for Single-Case Research: Nonoverlap of All Pairs. Behavior Therapy, 40(4), 357-367. https://doi.org/10.1016/j.beth.2008.10.006 
Peterson, C. L., \& Zettle, R. D. (2009). Treating inpatients with comorbid depression and alcohol use disorders: A comparison of Acceptance and Commitment Therapy and treatment as usual. The Psychological Record, 59, 521-536. https://doi.org/10.1007/BF03395679

Peterson, P. L., Baer, J. S., Wells, E. A., Ginzler, J. A., \& Garrett, S. B. (2006). Short-term effects of a brief motivational intervention to reduce alcohol and drug risk among homeless adolescents. Psychology of Addictive Behaviors, 20(3), 254.

https://doi.org/10.1037/0893-164X.20.3.254

Polk, K. L., Schoendorff, B., Webster, M., \& Olaz, F. O. (2016). The essential guide to the ACT matrix: A step-by-step approach to Using the ACT matrix model in clinical practice. New Harbinger Publications.

Ruscio, J. (2008). A probability-based measure of effect size: Robustness to base rates and other factors. Psychological Methods, 13(1), 19-30. https://doi.org/10.1037/1082-989X.13.1.19

Ruscio, J., \& Mullen, T. (2012). Confidence intervals for the probability of superiority effect size measure and the area under a receiver operating characteristic curve. Multivariate Behavioral Research, 47(2), 201-223. https://doi.org/10.1080/00273171.2012.658329

Scambler, G. (1998). Stigma and disease: changing paradigms. The Lancet, 352(9133), 1054-1055. https://doi.org/10.1016/S0140-6736(98)08068-4

Scott, J. (1993). Homelessness and mental illness. The British Journal of Psychiatry, 162(3), 314-324. https://doi.org/10.1016/S0140-6736(98)08068-4

Starr, S., Campbell, L. R., \& Herrick, C. A. (2002). Factors affecting use of the mental health system by rural children. Issues in Mental Health Nursing, 23(3), 291-304. https://doi.org/10.1080/016128402753543027

Stergiopoulos, V., Mejia-Lancheros, C., Nisenbaum, R., Wang, R., Lachaud, J., O'Campo, P., \& Hwang, S. W. (2019). Long-term effects of rent supplements and mental health support services on housing and health outcomes of homeless adults with mental illness: extension study of the At Home/Chez Soi randomised controlled trial. The Lancet Psychiatry, 6(11), 915-925. https://doi.org/10.1016/S2215-0366(19)30371-2

Tangney, J. P., \& Dearing, R. L. (2003). Shame and guilt. Guilford Press.

Thas, O., De Neve, J., Clement, L., \& Ottoy, J.-P. (2012). Probabilistic index models. Journal of the Royal Statistical Society: Series B (Statistical Methodology), 74(4), 623-671. https://doi.org/10.1111/j.1467$\underline{9868.2011 .01020 . x}$

Thornicroft, G. (2008). Stigma and discrimination limit access to mental health care. Epidemiology and Psychiatric Sciences, 17(1), 14-19. https://doi.org/10.1017/S1121189X00002621

Timms, P., \& Balázs, J. (1997). ABC of mental health: mental health on the margins. $B M J$, 315(7107), 536-539. https://doi.org/10.1136/bmj.315.7107.536

Tran, L., \& Rimes, K. A. (2017). Unhealthy perfectionism, negative beliefs about emotions, emotional suppression, and depression in students: A mediational analysis. Personality and Individual Differences, 110, 144-147. https://doi.org/10.1016/j.paid.2017.01.042

Trull, T. J., \& Ebner-Priemer, U. (2014). The role of ambulatory assessment in psychological science. Current Directions in Psychological Science, 23(6), 466-470. https://doi.org/10.1177/0963721414550706

Urbaniak, G. C., \& Plous, S. (2013). Research randomizer, Version 4.0. www.randomizer.org

Viechtbauer, W. (2010). Conducting Meta-Analyses in $\mathrm{R}$ with the metafor Package. Journal of Statistical Software, 36(3). https://doi.org/10.18637/jss.v036.i03

Webb, C. A., DeRubeis, R. J., \& Barber, J. P. (2010). Therapist adherence/competence and treatment outcome: A meta-analytic review. Journal of Consulting and Clinical Psychology, 78(2), 200. https://doi.org/10.1037/a0018912

Woolfolk, R. L. (2015). The value of psychotherapy: The talking cure in an age of clinical science. Guilford.

Ximenes, V. M., Manolov, R., Solanas, A., \& Quera, V. (2009). Factors Affecting Visual Inference in Single-Case Designs. The Spanish Journal of Psychology, 12(02), 823-832. https://doi.org/10.1017/S1138741600002195

Yadavaia, J. E., \& Hayes, S. C. (2012). Acceptance and commitment therapy for self-stigma around sexual orientation: A multiple baseline evaluation. Cognitive and Behavioral Practice, 19(4), 545-559. https://doi.org/10.1016/j.cbpra.2011.09.002

Zavaleta, D. (2015). Shame, humiliation and social isolation: Missing dimensions of poverty and suffering analysis. In R. E. Anderson's World Suffering and Quality of Life (pp. 251-266). Springer. 